\title{
Establishment of a green fluorescent protein tracing murine model focused on the functions of host components in necrosis repair and the niche of subcutaneously implanted glioma
}

\author{
ZHAO-HUI LU ${ }^{1 *}, \mathrm{KE} \mathrm{LV}^{2 *}$, JIN-SHI ZHANG ${ }^{1}$, CHUN-GANG DAI $^{3}$, BIN LIU $^{3}$, XIAO-YU MA ${ }^{3}$, \\ LIN-MING HE ${ }^{3}$, JING-YUN JIA ${ }^{3}$, YAN-MING CHEN ${ }^{1}$, XING-LIANG DAI ${ }^{1}$, AI-DONG WANG ${ }^{1}$, \\ JUN DONG $^{1}$, QUAN-BIN ZHANG ${ }^{2}$, QING LAN ${ }^{1}$ and QIANG HUANG ${ }^{1}$ \\ ${ }^{1}$ Neurosurgical Department of The Second Affiliated Hospital, Soochow University, Suzhou 215004; \\ ${ }^{2}$ Neurosurgical Department of Shanghai Tenth People's Hospital, Tongji University, Shanghai 200072; \\ ${ }^{3}$ Neurosurgical Department of Suzhou Seventh People's Hospital, Suzhou 215004, P.R. China
}

Received August 14, 2013; Accepted September 13, 2013

DOI: $10.3892 /$ or.2013.2873

\begin{abstract}
Due to progress in the research of glioma stem cells and the glioma niche, development of an animal model that facilitates the elucidation of the roles of the host tissue and cells is necessary. The aim of the present study was to develop a subcutaneous xenograft green fluorescent protein nude mouse model and use this model to analyze the roles of host cells in tumor necrosis repair. Tumors derived from the human glioma stem/progenitor cell line SU3 were subcutaneously implanted in green fluorescent protein nude mice. The implanted tumors were then passed from animal to animal for 10 generations. Finally, subcutaneous xenografts were assayed with traditional pathology, immunopathological techniques and fluorescence photography. For each generation, the tumorigenicity rate was $100 \%$. Subcutaneous xenografts were rich in blood vessels, and necrotic and hemorrhagic foci, which highly expressed hypoxia-inducible factor- $1 \alpha$, tumor necrosis factor, Ki-67, CD68 and CD11b. In the interstitial tissue, particularly in old hemorrhagic foci, there were numerous cells expressing green fluorescent protein, CD68 and CD11b. Green fluorescent protein nude mouse subcutaneous xenografts not only consistently maintained the high invasiveness and tumorigenicity
\end{abstract}

Correspondence to: Dr Quan-Bin Zhang, Neurosurgical Department of Shanghai Tenth People's Hospital, Tongji University, 301 Yanchang Road, Shanghai 200072, P.R. China

E-mail: quanbinzhang@yahoo.com.cn

Dr Jun Dong, Neurosurgical Department of The Second Affiliated Hospital, Suzhou University, 181 Sanxiang Road, Suzhou 215004, P.R. China

E-mail: jundong456@yeah.net

${ }^{*}$ Contributed equally

Key words: tumor microenvironment, cancer stem cell, tumor necrosis and repair, green fluorescent protein nude mice of glioma stem/progenitor cells, but also consisted of a high concentration of tumor blood vessels and necrotic and hemorrhagic foci. Subcutaneous xenografts also expressed high levels of tumor microenvironment-related proteins and hostderived tumor interstitial molecules. The model has significant potential for further research on tumor tissue remodeling and the tumor microenvironment.

\section{Introduction}

The most common type of brain tumor in adults is glioma, which is extraordinarily lethal and is associated with extremely low survival rates $(1,2)$. For most malignant forms of glioma, the median overall survival is only $12-15$ months (2). The most common as well as the most aggressive type of glioma is glioblastoma multiforme (1). Glioblastoma multiforme consists of heterogeneous types of cells which demonstrate a variety of tumorigenic properties (1).

Gliomas arise from neural stem cells and progenitors (1). These neural stem cells are a subtype of astrocytes and are located in the two main regions of the brain where neurogenesis takes place: the subventricular region and the subgranular zone of hippocampal formation $(2,3)$.

After cancer cells begin to propagate, they construct their own microenvironment or niche (1). In this niche there is 'crosstalk' between the tumor and the host so that the niche and its surroundings constantly interact $(1,4)$.

One condition that promotes tumor growth is hypoxia (5). Rapid growth of a tumor that outpaces neovascularization results in a hypoxic niche (1). Hypoxia-inducible factor 1 (HIF-1) is an important regulator of cellular responses to hypoxia (5). HIF-1 promotes glioma growth, mainly by activating angiogenesis and by upregulating relevant target genes (5).

Animal models are critical for investigating malignant tumors. The orthotopic xenograft mouse model of glioblastoma has been particularly valuable for analyzing the characteristics of donor human tumor cells, but studies concerning the role of host tissue and cells are sparse (6). Due to the progress 
achieved in the research of cancer stem cells and the tumor microenvironment (7), it has become obviously necessary to have a comprehensive understanding of the role of host-derived tissues and cells in tumor development. In recent years, green fluorescent protein (GFP) nude mice have been widely adopted for human cancer xenograft models with the main focus on the tracing of tumor cells in the xenografts $(8,9)$. The aims of the present study were to develop a subcutaneous xenograft GFP nude mouse model by injection of human glioma stem/progenitor cell line SU3, and investigating the potential roles of host cells in the repair of necrosis in xenografts. We successfully established a model that facilitates the observation of how host cells participate in the repair of tumor necrosis.

\section{Materials and methods}

Materials. The human glioma stem/progenitor cell line SU3 was established according to previously published methods from a surgical specimen of an adult male patient diagnosed with glioblastoma multiforme (10). In the present study, the human glioma stem/progenitor cell line SU3 was successfully obtained from fresh surgical specimens of GBM patients. These patients underwent surgery at the Neurosurgical Department, Second Affiliated Hospital of Suzhou University. The present study was approved by the Medical Review Board of the Suzhou University Medical School. The patients or their legal guardians provided informed consent for the use of their surgical specimen in research prior to sample acquisition. GFP nude NC-C57BL/6J mice were bred and maintained in a specific pathogen-free animal care facility. Animal experiments were approved by the Medical Review Board of Suzhou University Medical School, and all procedures were conducted in accordance with Chinese laws governing animal care. The trocars, namely \#8 anesthesia epidural puncture needles (Zhejiang Haisheng Medical Instrument Co., Ltd., Hangzhou, China) were selected for the passage of tumor tissue in vivo. A fluorescence flashlight and fluorescence glasses were provided by NightSea Co. (http://www.nightsea.com/fplights.htm). The frozen sections of the xenografts were observed by fluorescence microcopy (Carl Zeiss Axio Observer A1; Thornwood, New York, USA). Immunohistochemical antibodies against TNF-1 $\alpha$ (ab1793), HIF-1 $\alpha$ (ab8366), Ki-67 (ab92742), CD68 (ab53444) and CD11b (ab64347) were purchased from Abcam (Cambridge, UK).

Tumor xenograft model. The human glioma stem/progenitor cell line SU3 was successfully obtained from fresh surgical specimens of GBM patients. SU3 cells were cultured in Dulbecco's modified Eagle's medium (DMEM) with $10 \%$ fetal bovine serum (FBS) and incubated at $37^{\circ} \mathrm{C}$ in $98 \%$ humidified air containing $5 \% \mathrm{CO}_{2}$. The SU3 cells in logarithmic growth phase in vitro were digested into a single-cell suspension liquid, and the final cell density was adjusted to $1 \times 10^{5} / \mathrm{ml}$. A cell suspension, $150 \mu \mathrm{l}$ per mouse, was slowly and smoothly injected subcutaneously into the right forelimb axilla of the GFP nude mice. The implanted tumor tissue was dissected free of blood clots, washed and minced into $0.5-\mathrm{mm}$ slices for tumor tissue grafting. For the tumor tissue transplantation, we designed a simple but novel injection system, which included a $24 \#$ trocar and a specially designed propeller (1). The tumor tissue, $1 \mathrm{~mm}^{3}$ per mouse, was slowly and smoothly injected into the right forelimb axilla of the GFP nude mice. The animal model established through tumor tissue injection was regarded as the first generation in the present study. The implanted tumors were passed from animal to animal following the same procedure for 10 generations, which was the point at which model stability was established. The short diameter (a) and long diameter (b) of the xenografts were measured with calipers every 3 days, and the tumor volume was calculated according to the equation: $\mathrm{V}\left(\mathrm{mm}^{3}\right)=\mathrm{a}^{2} \mathrm{x} b / 2$. When cachexia occurred, the tumor-bearing mice were sacrificed for tumor removal and examination.

Molecular pathology and GFP expression of the tumors. We performed immunohistochemical staining to examine the expression of tumor necrosis factor (TNF) (1:50), hypoxiainducible factor $1 \alpha$ (HIF-1 $\alpha)$ (1:200), Ki-67 (1:1,000), CD68 (1:200) and CD11b (1:400) in the xenografts (1). The tumorbearing mice and the subcutaneous xenografts were examined with a fluorescence flashlight and fluorescence glasses. Cells expressing GFP in the tumor tissue were examined using 5- $\mu \mathrm{m}$ frozen sections by fluorescence microscopy at a wavelength of $470 \mathrm{~nm}$.

\section{Results}

General characteristics of the xenografts. In the primary xenograft models (SU3 cell suspension injection), although the tumorigenicity rate was $100 \%(5 / 5)$, there existed obvious individual differences in incubation period and tumor volume. In conjunction with the tumor passage through tumor tissue injection, these differences gradually narrowed, and the animal model became reliable beginning with the 7th generation. Subcutaneous seeded tumors can grow quite large; the xenograft volume of the 9th generation, one month after inoculation, was $\sim 9.96 \pm 4.19 \mathrm{~cm}^{3}(\mathrm{n}=10)$ in average and the implanted tumors continued to grow for more than 2 months. When all the tumor-bearing mice were sacrificed in the late stage (approximately 2 months after tumor inoculation), the tumor weight reached almost one half of the body weight of the mice, namely, $11.23 \pm 3.72 \mathrm{~g}(\mathrm{n}=10)$.

Under a fluorescence flashlight, the tumor-bearing mice and the xenografts appeared green in color (Fig. 1A and B). Viewed with the naked eye under natural light, there was enlargement of the xenografts, and cutaneous vessels of the skin covering tumors were gradually increased in size, meeting the demand of the blood supply required by the rapidly growing tumor tissue (Fig. 1C and D). In the middle stage of xenograft growth, ischemic necrosis, even to the extent of ulceration, was observed in the local skin, possibly indicating the insufficiency of the blood supply to the implanted tumors (Fig. 1E); there was still fresh tissue in the tumor base (Fig. 1F).

Histopathologic characteristics of the xenografts. In the xenograft tumor sections stained with hematoxylin and eosin (H\&E), hyperchromatic nuclei, relatively less cytoplasm, heteromorphic nuclei and regional densely arranged tumor cells were observed (Fig. 2). Necrotizing hemorrhage was frequently observed in the xenograft sections and was divided 

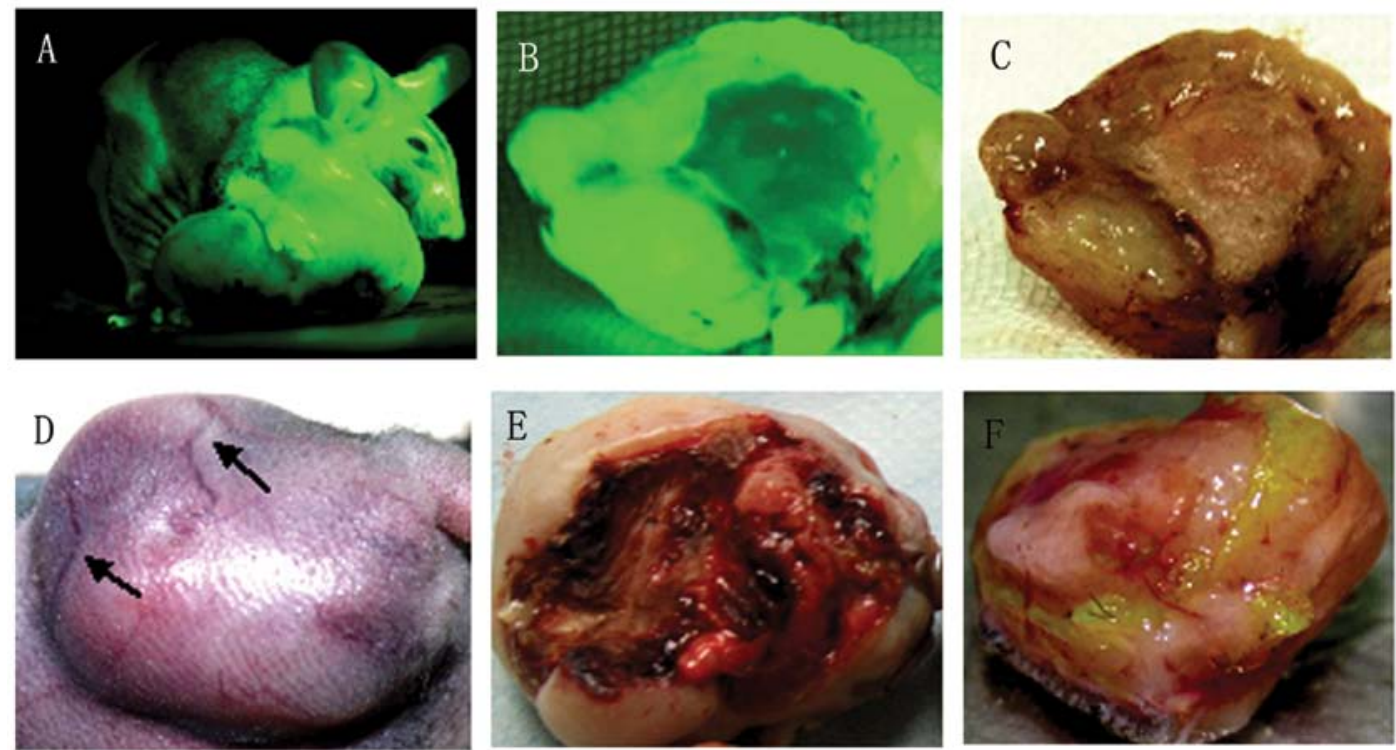

Figure 1. Gross anatomy of a transplanted tumor. Under the illumination of a fluorescence flashlight, (A) the external appearance and (B) the central section of the tumor in the right subaxillary region of a tumor-bearing mouse exhibit green fluorescence. (C) Under natural light, the peripheral region of the central section of the tumor exhibits a light green color, and the center has a fresh meat-like appearance (image of the same specimen as B). (D) In the early stage, engorgement of blood vessels is observed on the skin surface of the tumor (arrows). (E) In the middle and late stages, a large area of necrotic scabs and ulcers following decrustation are noted. (F) However, the tissue in the tumor base was still fresh.
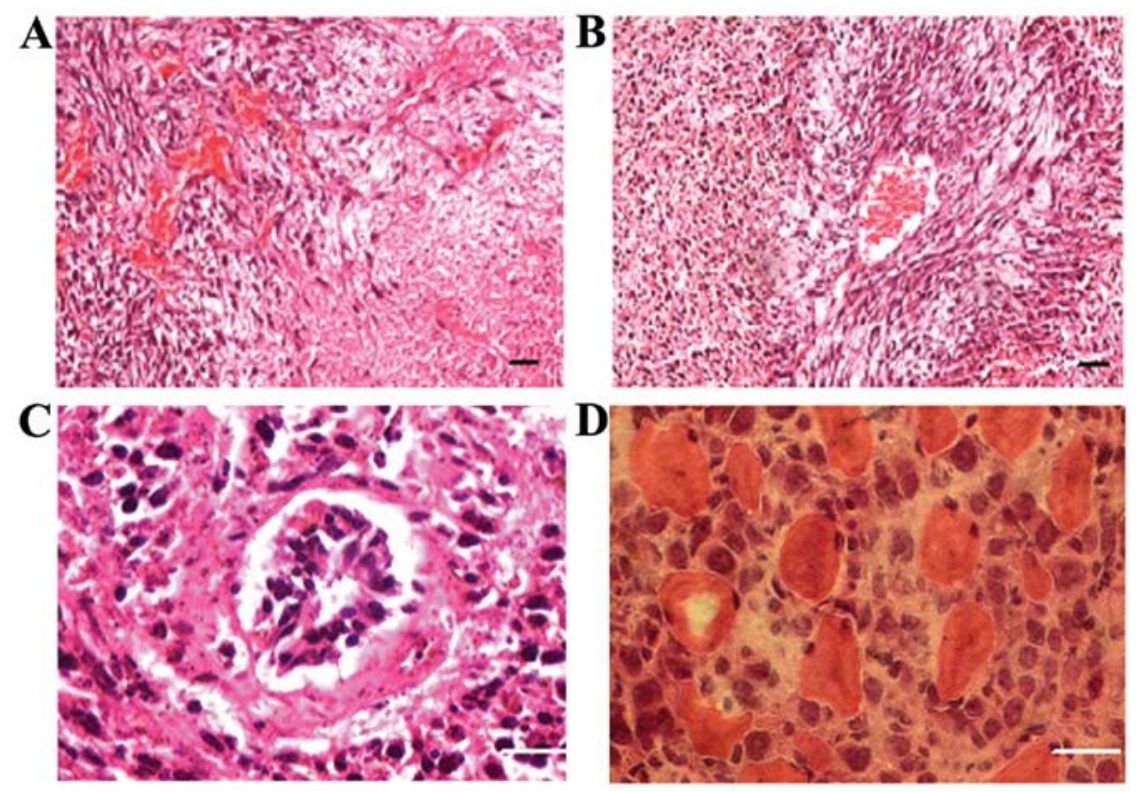

Figure 2. Paraffin-embedded H\&E-stained sections. (A) Pleomorphic (mainly spindle-shaped) cells are densely arranged, blood vessels are abundant and are shown as blood sinus, and hemorrhagic necrosis is commonly noted. (B) Proliferation of plentiful fibroblast-like cells is occasionally observed around the blood vessels in the old necrotic region. (C) Inside the blood vessels, tumor thrombi composed of tumor cells are occasionally observed. (D) Tumor cells were found to extensively invade the soft tissue and the muscle fiber. Scale bar, $25 \mu \mathrm{m}$.

into fresh hemorrhage and old hemorrhage (Fig. 2A). In the fresh hemorrhagic foci, a large number of collapsing red blood cells could still be observed. In the old hemorrhagic areas, two scenes were observed: i) red blood cells disappeared while some inflammatory cells were observed; and ii) there were no inflammatory cells, however, tumor cells with relatively few mesenchymal components were noted growing around the vessels. Generally, the xenograft tumors were rich in blood vessels, but a vast majority of new blood vessels lacked complete vascular structure. Only a few large vessels possessed a nearly intact vascular wall. In rare instances, clusters of nucleated cells were noted in the vascular lumen. Tumor cells could be seen invading soft tissue and muscle fiber (Fig. 2D).

Molecular pathology of the xenografts. Hypoxia-inducible factor $1 \alpha$ was strongly expressed in the nuclei of the tumor cells (Fig. 3A and B). Generally, the expression of HIF-1 $\alpha$ was 

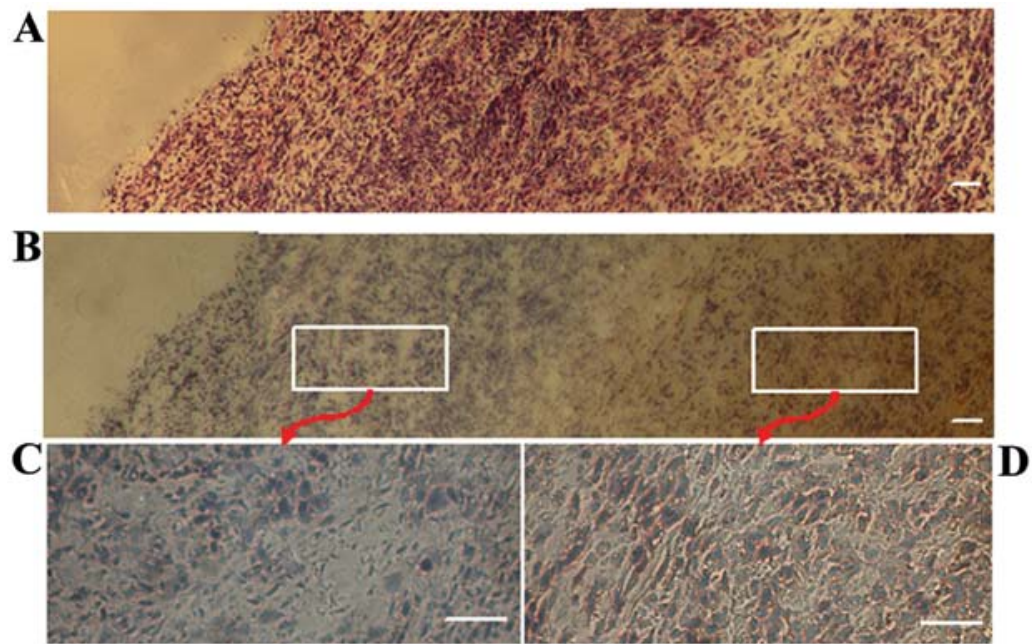

Figure 3. Different levels of HIF- $\alpha$ expression are noted in the center and margin of tumors. (A and B) Adjacent sections of the same specimen. H\&E staining and immunohistochemical staining for HIF- $\alpha$ were performed, respectively. The tumor margin extends to the center with a left-to-right direction. (C and D) Magnified images of the tumor margin and the central frame. HIF- $\alpha$ expression in the tumor center is significantly stronger than that in the tumor margin. Scale bar, $25 \mu \mathrm{m}$.

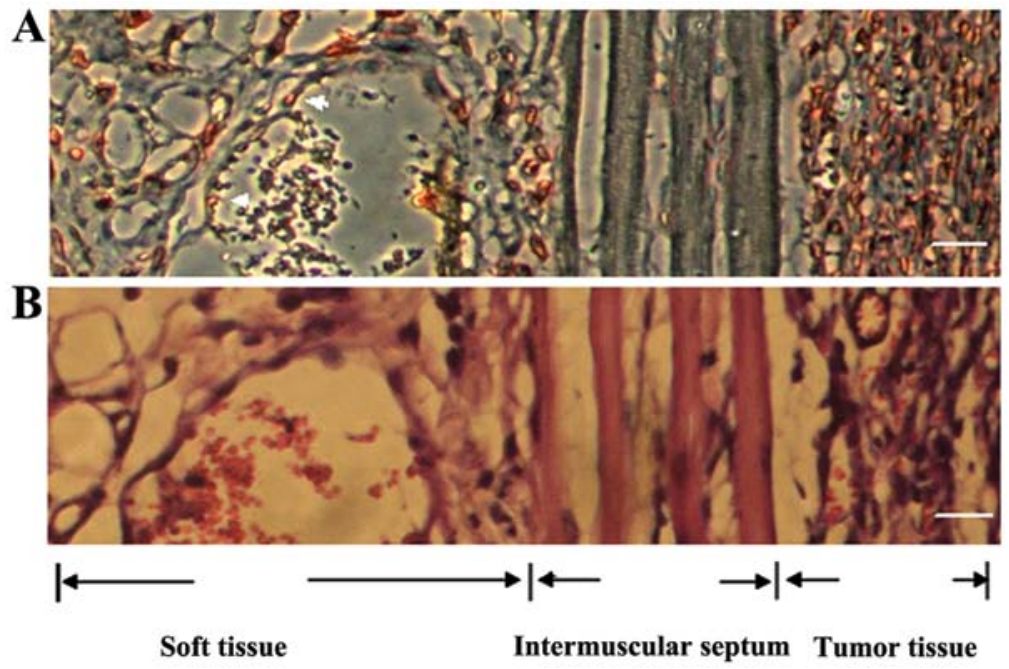

Figure 4. HIF- $\alpha$ is expressed in invading tumor cells. (A and B) Adjacent sections of the same specimen. H\&E staining and immunohistochemical staining for HIF- $\alpha$ were performed, respectively. From left to right are the subcutaneous soft tissue, muscle and tumor entity. HIF- $\alpha$ is highly expressed not only in the cells inside the tumor entity, but also in the tumor cells invading the subcutaneous soft tissue and blood vessel walls (white arrow). Scale bar, $100 \mu \mathrm{m}$.

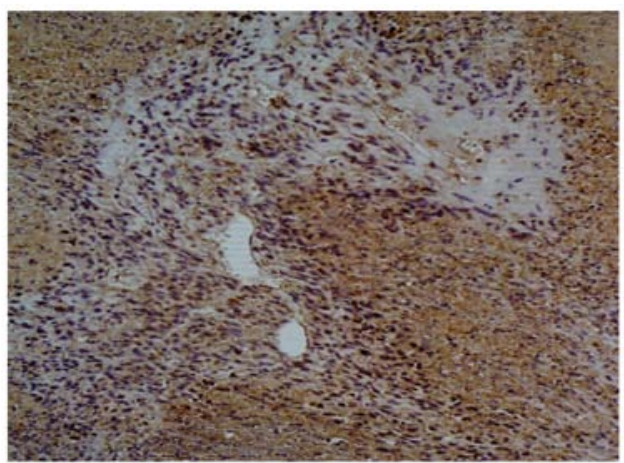

Figure 5. Expression of TNF in the tumor tissue (immunohistochemical staining; magnification, $\mathrm{x} 400$ ). Brown immune complexes are mainly distributed in the region of obvious tumor necrosis, and they are deposited in the intercellular substance. Although the cytoplasm of the tumor cells in the non-necrotic region also contain these types of complexes, the frequency is less significant compared to the necrotic region. high in the central portion of the tumor and low in the marginal zones (Fig. 3C and D); however, notably, invasive tumor cells migrating from the marginal zones also highly expressed HIF-1 $\alpha$ (Fig. 4). In the necrotic areas of the tumors, TNF was mainly expressed in shapeless intercellular fluid; however, in the non-necrotic areas of the tumors, TNF was diffusedly expressed on the membrane of tumor cells rather than in the intercellular fluid (Fig. 5). The vast majority of tumor cells, regardless of cell type, including those in the necrotic areas, strongly expressed Ki-67 in the nuclei (Fig. 6). In old hemorrhagic areas, the majority of cells were positively stained for CD11b and CD68, which were expressed both in the cytoplasm and on the membrane (Fig. 7).

Green fluorescence components in the xenografts. As the host organs, tissues and cells were GFP-expressing, all 

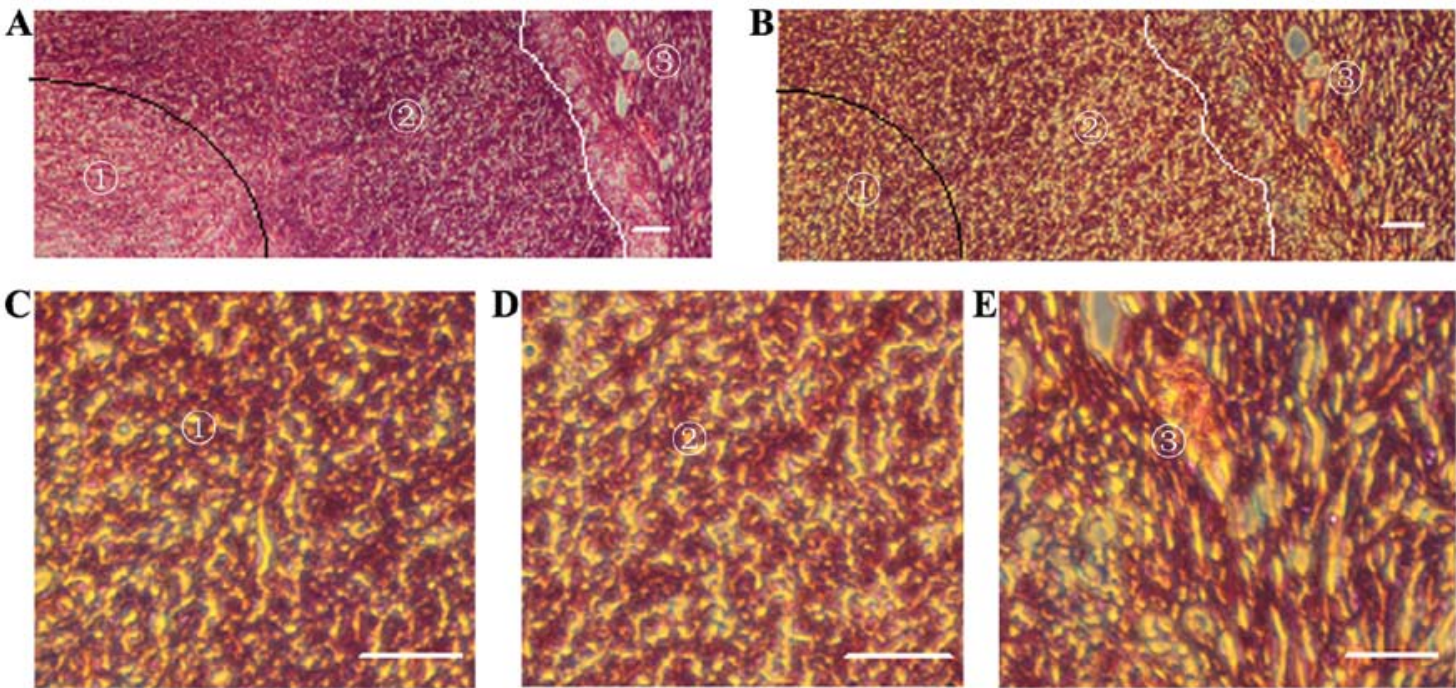

Figure 6. Immunohistochemical staining for Ki-67. (A and B) Adjacent sections of the same specimen. In the H\&E-stained section (A), the old necrotic region (1), the marginal region around the necrosis (2), and the region with active tumor proliferation and rich in blood vessels (3) are clearly observed. (C-E) Magnified images of (1), (2) and (3) from B. Ki-67 expression in the nucleus was found to be highly positive in all cells. However, analysis of cell morphology revealed that (1) and (2) mainly include small round cells and (3) mainly includes spindle cells. Scale bar, $50 \mu \mathrm{m}$.

A

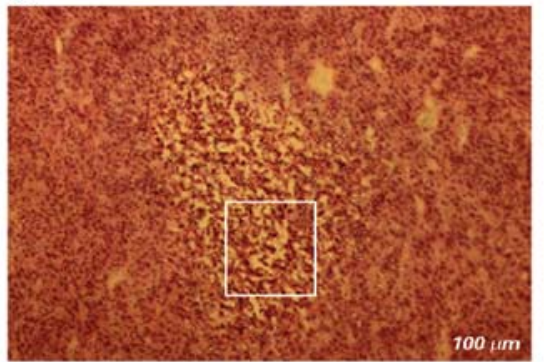

C

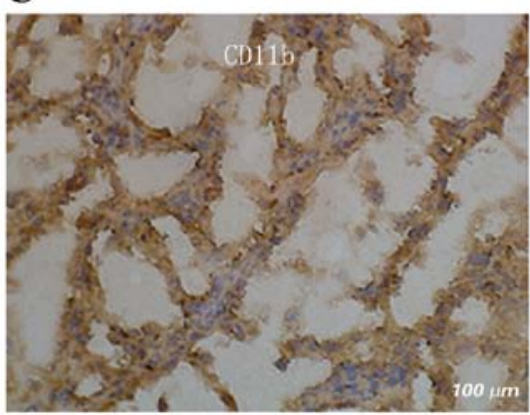

B

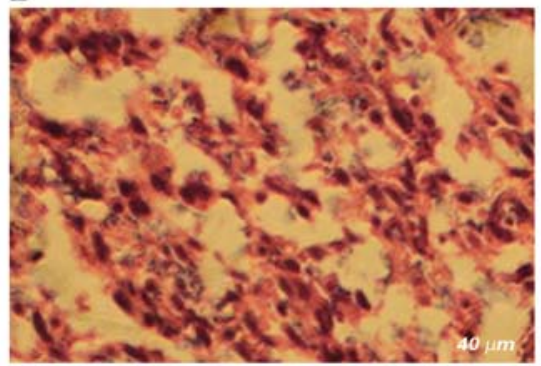

D

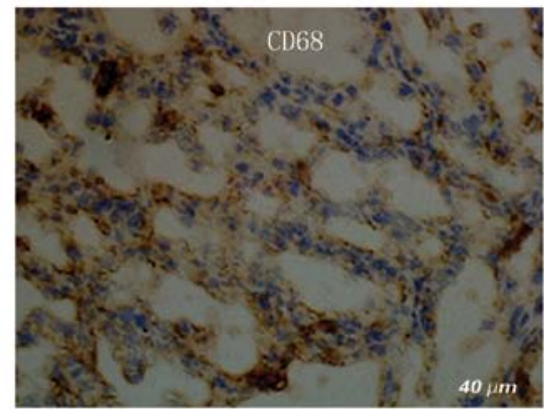

Figure 7. Inflammatory cell infiltration in the old necrotic region. There is an absence of intercellular substance in the H\&E-stained section. (A) Cells are arranged relatively sparsely in the old necrotic region. (B) Magnified image of A; magnification, $\mathrm{x} 400$. (C) CD11b and (D) CD68 are highly expressed in cells of the immunohistochemically stained adjacent sections. Scale bar, $50 \mu \mathrm{m}$.

components in the xenografts displayed green fluorescence under fluorescence microscopy at an excitation wavelength of $470 \mathrm{~nm}$ and were regarded to be derived from the host. At the same time, tumor cells did not stimulate green fluorescence under fluorescence microscopy at an excitation wavelength of $470 \mathrm{~nm}$. Based on this concept, it was obvious that the implanted tumors were covered by host-derived skin, and the green area surrounding the tumor mass mainly consisted of host components (Fig. 1A and B). Notably, a large number of GFP-expressing cells were found clustered in the necrotic areas of the tumors (Fig. 8). GFP-expressing cells that had a similar appearance to macrophagocytes were also found scattered among the tumor parenchyma (Fig. 9).

\section{Discussion}

In the present study, we developed an animal model for studying the role of host cells in glioma. We used a subcutaneous xenograft GFP nude mouse model and passed implanted tumors from animal to animal for 10 generations. The xenografts, which came from the human glioma stem/progenitor cell line SU3, were rich in blood vessels, necrosis and hemorrhagic foci. 

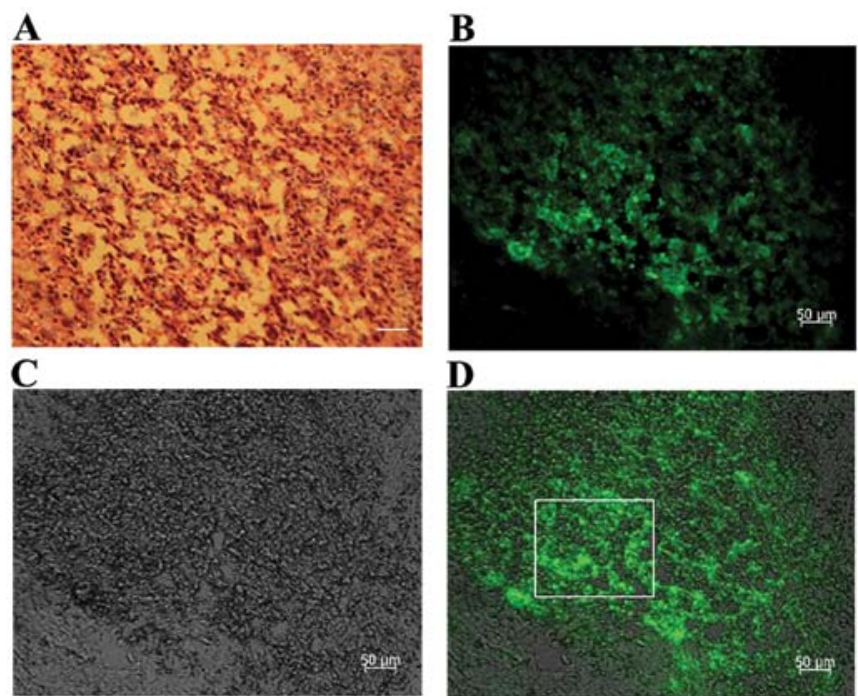

$\mathbf{E}$

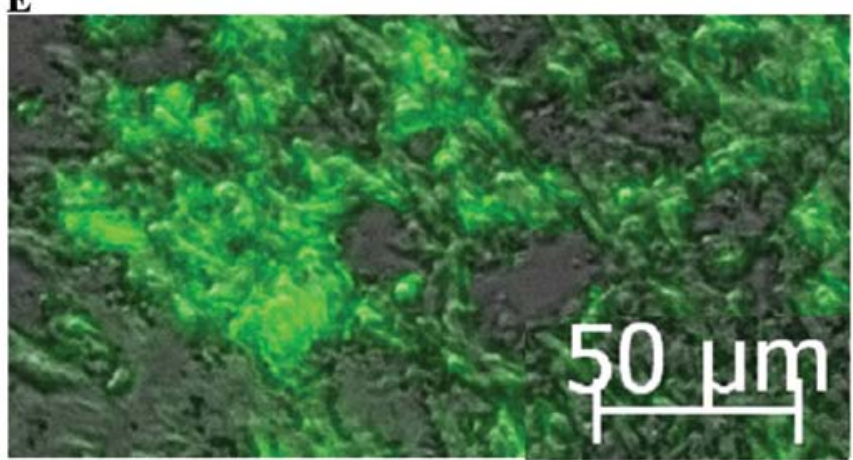

Figure 8. Microenvironment of the necrotic region in the tumor as shown by green fluorescent protein (GFP). (A and B) Adjacent sections of the same specimen. Following H\&E staining, sparsely arranged cells in the old necrotic region can be observed under an ordinary microscope and interstitial components are rare (A). Relatively numerous GFP components can be observed in the necrotic region under a fluorescence microscope. (C) The tumor cells do not express GFP, which are shown as grayish-black under non-excitation light. (D) Overlapping image of B and C. The GFP component is embedded in the tumor stroma to be part of the microenvironment. (E) Magnified image of D. These types of GFP components can be divided into amorphous substances and cells with different shapes. Scale bar, $50 \mu \mathrm{m}$.

In the interstitial tissue of the host, we noted numerous cells expressing GFP, CD68 and CD11b. Based on our findings we believe this model has significant potential for application in the future research of tumor tissue remodeling and the tumor niche.

Hypoxia inducible factor $1 \alpha$ (HIF-1 $\alpha$ ). Hypoxia-inducible factor 1 plays an important role in tumor growth by being a key regulator of cellular responses to hypoxia $(5,11,12)$. It consists of $\alpha$ and $\beta$ subunits (5). In hypoxic conditions, the $\alpha$ subunit is stable and in normoxia it is rapidly degraded (5). HIF-1 is translocated to the cell nucleus and induces transcription of its downstream target genes such as vascular endothelial growth factor and erythropoietin.

Hypoxia-inducible factor 1 has been reported to play an important role in tumor growth and metastasis by regulating energy metabolism and inducing angiogenesis to allow survival in cellular hypoxia. Increased levels of HIF-1 have been noted in breast carcinogenesis, lung cancer, prostatic
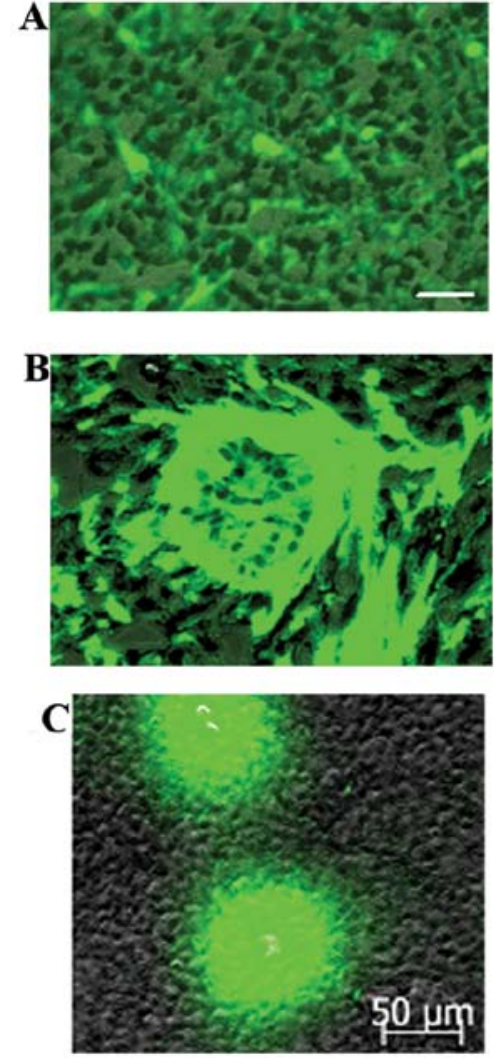

Figure 9. Image of overlapping fluorescence light and white light. The tumor cells are shown in black, and the host tissue and cells are green in color. (A) Host cells are noted within the tumor entity. (B) Tumor cells are noted which enter the host blood vessel to form a tumor thrombus. (C) Wave-shaped macrophages on the cell membrane are noted. Scale bar, $50 \mu \mathrm{m}$.

cancer, gastric carcinoma and cervical cancer (13-16). Therefore, it was necessary to evaluate the expression of HIF-1 in the assessment of our animal model. HIF-1 expression in our tumor model showed two features: i) HIF-1 $\alpha$ was highly expressed in the central portion of the tumor and expression was low in the tumor margin which was mainly due to low oxygen conditions in the central portion of the tumor (17); and ii) tumor cells invading the host tissue where oxygen is sufficient also highly expressed HIF-1. We conjecture that the high expression of HIF-1 in non-hypoxic host tissue was associated with the disorder of tumor-suppressor genes (18). With regard to expression of HIF-1, this tumor animal model meets the required criterion.

Expression of tumor necrosis factor (TNF). Tumor necrosis factor was originally discovered in hemorrhagic necrotic tissue removed from tumor-bearing mice. TNF, which consists of TNF- $\alpha$ and TNF- $\beta$, was found to be secreted by macrophages and leukomonocytes. TNF- $\alpha$ is a type II membrane protein and contains soluble TNF- $\alpha$ and membrane-related TNF- $\alpha$. The effect of TNF- $\alpha$ is concentration-dependent and type-dependent, including the induction of tumor cell apoptosis (19) and immune surveillance (20) or the effects are opposite depending on dose. For example, at high doses, TNF exerts a degenerative effect on tumor vasculature and induces tumor hemorrhage and necrosis; at moderate doses, TNF does not have cytotoxic effects on tumor cells, but 
stimulates the proliferation and metastasis of tumor cells by inducing angiogenesis and promoting secretion of matrix metalloproteinase and endothelial adhesion molecule $(21,22)$. In tumor necrotic areas of our animal model, TNF appeared to have the characteristic of STNF- $\alpha$; it was highly expressed in shapeless intercellular fluid, distributed lamellarly and without specific cell morphology. As there were numerous immune cells infiltrating into tumor necrotic areas, sTNF- $\alpha$ was most likely secreted by these immune cells (Fig. 8). Nevertheless, in the non-necrotic areas of the tumors, TNF appeared to have the characteristic of mTNF- $\alpha$; it was diffusely expressed on the membrane of tumor cells. For this reason, mTNF- $\alpha$ was possibly secreted by tumor cells. In brief, the expression of TNF- $\alpha$ in this subcutaneous GFP nude mouse model conformed to the features of malignant tumors.

Tumor microenvironment. The tumor microenvironment, also called the tumor niche, is composed of interstitial fluid and various types of cells (inflammatory cells, endothelial cells and fibroblasts). It is known that in the early stage, the host-derived microenvironment exerts suppressive effects on the growth of transplanted tumors due to transplant rejection; however, in conjuction with the growth of transplanted tumors, the microenvironment changes in favor of tumor growth. This phenomenon has become a topic of extreme importance in oncology research during the last few decades. In the early years of research, Bettinger et al (23) reported that microglia can promote glioma migration and Markovic et al (24) reported that when microglia were selectively depleted, the invasiveness of tumors was significantly decreased. In recent years, Wu et al (25) reported that macrophages/microglia have the potential to promote the proliferation of tumor cells. Straussman et al (26) reported that when co-cultured with stromal cells, tumor cells become more resistant in vitro to chemotherapy. Taken together, these studies indicate that the host-derived tumor microenvironment plays a critical role in tumor development and progression. In another in vitro study of the tumor microenvironment, Borovksi et al (27) used a co-culture system comprising primary brain tumor microvascular endothelial cells and glioma propagating cells obtained from glioblastoma multiforme biopsies. They found that glioma propagating cells had higher proliferation rates when co-cultured with primary brain tumor microvascular endothelial cells than when cultured alone. However, in vitro experiments are lacking in the ability to demonstrate the importance of the tumor microenvironment.

In vivo experiments were performed by Najbauer et al (4) who used orthotopic rodent models of human glioma xenografts and reported that a large number of host cells expressing nestin migrate to glioma and contribute to the growth of the xenograft by assembling into the microvasculature of glioma. It was determined that the host cells originated from the subventricular zone ipsilateral to the xenografts. The advantage of the GFP nude mouse model established in the present study is that it is convenient for tracing host cells and investigating the role of host cells in tumor growth. For example, a large amount of GFP-expressing cells were found clustered in old necrotic areas, and the majority of cells expressed CD11b and CD68, which are inflammatory cells that can serve as markers for macrophage-specific cells. Thus, we deduced that host cells are critical component of the tumor microenvironment that play a role in the repair of tumor necrosis. As determined by the immunochemical staining, numerous TNF- $\alpha$ immune complexes were noted in the necrotic areas of the tumors (Fig. 5). This indicated that TNF- $\alpha$ secreted by macrophages participates in the composition of the cell factor microenvironment. A large number of cells expressing Ki-67 was noted in both the tumor necrotic areas and non-necrotic areas (Fig. 6). Ki-67 is a nuclear proliferation antigen and its expression reflects the proliferative ability of cells, and for tumor cells its expression also predicts malignancy and poor prognosis. Since Ki-67-expressing cells included GFP cells, this added more evidence to support the assumption that hostderived cells are involved in the repair of tumor necrosis. Due to the participation of host cells in the repair of necrosis of tumor tissue, no cyst was discovered in our more than 100 tumor specimens.

de Almeida Sassi et al (1) recently reviewed studies on glioma including the extensive research that has been carried out to describe the tumor microenvironment. Importantly, they revealed that there is a reciprocal relationship between glioma stem cells and their microenvironment; that is, glioma stem cells can modulate the microenvironment that produces signals that regulate glioma stem cells. They also pointed out that tumor microvasculature generates niches that promote the establishment and maintainence of brain tumor stem cells. In addition, niches were found to have an important role in protecting these stem cells from environmental insults (e.g., chemotherapeutic agents). Recently, Gazdzinski and Neiman (28) injected GL261 and 4C8 glioma cells that were labeled with iron oxide particles or with a fluorescent probe into the brains of synergeneic mice. Following tumor development, they used texture analysis to analyze the label distribution patterns in the two types of gliomas. They reported that in the GL261 tumors the label was observed mainly in the tumor core whereas in the 4C8 tumors the label was more randomly distributed throughout the tumor. They speculated that differences in the label distribution maps between the two different types of gliomas were likely affected by differences in the tumor microenvironment.

In conclusion, a glioma model consisting of GFP nude mice as the host was successfully established. With this model, we were easily able to observe how host cells participate in tumor necrosis repair. Since the host cells constantly express GFP, this model has great potential for investigating the tumor microenvironment as a supporting role in tumor growth and progression and may serve to identify future therapeutic targets.

\section{Acknowledgements}

The present study was funded by the National Natural Scientific Foundation of China (nos. 81071766; 81172400; 81272799; and 81272793).

\section{References}

1. de Almeida Sassi F, Brunetto AL, Schwartsmann G, Roesler R and Abujamra AL: Glioma revisited: from neurogenesis and cancer stem cells to the epigenetic regulation of niche. J Oncol 2012: 537861, 2012. doi:10.1.1155/2012/537861. 
2. He H, Li MU and Niu CS: The pathological characteristics of glioma stem cell niches. J Clin Neurosci 19: 121-127, 2012.

3. Tavazoie M, Van der Veken L, Silva-Vargas V, et al: A specialized vascular niche for adult neural stem cells. Cell Stem Cell 3: 279-288, 2008.

4. Najbauer J, Huszthy PC, Barish ME, et al: Cellular host responses in gliomas. PLoS One 7: e35150, 2012.

5. Kaur B, Khwaja FW, Severson EA, Matheny SL, Brat DJ and Van Meir EG: Hypoxia and the hypoxia-inducible-factor pathway in glioma growth and angiogenesis. Neuro Oncol 7: 134-153, 2005.

6. Fei XF, Zhang QB, Dong J, et al: Development of a clinically relevant orthotopic xenograft mouse model of metastatic lung cancer and glioblastoma through surgical tumor tissue injection with trocar. J Exp Clin Cancer Res 29: 84, 2010.

7. Charles NA, Holland EC, Gilbertson R, Glass R and Kettenmann H: The brain tumor microenvironment. Glia 60 : 1169-1180, 2011.

8. Hoffman RM: Green fluorescent protein imaging of tumor cells in mice. Lab Animal 31: 34-41, 2002.

9. Farin A, Suzuki SO, Weiker M, Goldman JE, Bruce JN and Canoll P: Transplanted glioma cells migrate and proliferate on host brain vasculature: a dynamic analysis. Glia 53: 799-808, 2006.

10. Wan Y, Fei XF, Wang ZM, et al: Expression of miRNA-125b in the new highly invasive glioma stem cell and progenitor cell line SU3. Chin J Cancer 31: 207-214, 2012.

11. Gillespie DL, Whang K, Ragel BT, Flynn JR, Kelly DA and Jensen RL: Silencing of hypoxia inducible factor-1 $\alpha$ by RNA interference attenuates human glioma cell growth in vivo. Clin Cancer Res 13: 2441-2448, 2007.

12. Ryan HE, Poloni M, McNulty W, Elson D, Gassmann M, Arbeit JM and Johnson RS: Hypoxia-inducible factor- $1 \alpha$ is a positive factor in solid tumor growth. Cancer Res 60: 4010-4015, 2000.

13. Bos R,Van der Groep P, Greijer AE, et al: Levels of hypoxiainducible factor- $1 \alpha$ independently predict prognosis in patients with lymph node negative breast carcinoma. Cancer 97: 1573-1581, 2003

14. Zhong H, Semenza GL, Simons JW and De Marzo AM: Up-regulation of hypoxia-inducible factor $1 \alpha$ is an early event in prostate carcinogenesis. Cancer Detect Prev 28: 88-93, 2004.

15. Birner P, Schindl M, Obermair A, Plank C, Breitenecker G and Oberhuber G: Overexpression of hypoxia-inducible factor 10 is a marker for an unfavorable prognosis in early-stage invasive cervical cancer. Cancer Res 60: 4693-4696, 2000.
16. Urano N, Fujiwara Y, Doki Y, et al: Overexpression of hypoxiainducible factor-1 alpha in gastric adenocarcinoma. Gastric Cancer 9: 44-49, 2006.

17. Clottes E: Hypoxia inducible factor 1: regulation,involvement in carcinogenesis and target for anticancer therapy. Bull Cancer 92: 119-127, 2005 (In French).

18. Xia Y, Choi HK and Lee K: Recent advances in hypoxiainducible factor (HIF)-1 inhibitors. Eur J Med Chem 49: 24-40, 2012.

19. Lyu MA and Rosenblum MG: The immunocytokine scFv23/TNF sensitizes HER-2/neu-overexpressing SKBR-3 cells to tumor necrosis factor (TNF) via up-regulation of TNF receptor-1. Mol Cancer Ther 4: 1205-1213, 2005 .

20. Ebert LM, Meuter S and Moser B: Homing and function of human skin $\gamma \delta$ T cells and NK cells: relevance for tumor surveillance. J Immunol 176: 4331-4336, 2006.

21. Warren MA, Shoemaker SF, Shealy DJ, Bshara W and Ip MM: Tumor necrosis factor deficiency inhibits mammary tumorigenesis and a tumor necrosis factor neutralizing antibody decreases mammary tumor growth in neu/erbB2 transgenic mice. Mol Cancer Ther 8: 2655-2663, 2009.

22. Yin Y, Chen X and Shu Y: Gene expression of the invasive phenotype of TNF- $\alpha$-treated MCF-7 cells. Biomed Pharmacother 63: 421-428, 2009.

23. Bettinger I, Thanos S and Paulus W: Microglia promote glioma migration. Acta Neuropathol 103: 351-355, 2002.

24. Markovic DS, Glass R, Synowitz M, Rooijen Nv and Kettenmann H: Microglia stimulate the invasiveness of glioma cells by increasing the activity of metalloprotease-2. J Neuropathol Exp Neurol 64: 754-762, 2005.

25. Wu A, Wei J, Kong LY, et al: Glioma cancer stem cells induce immunosuppressive macrophages/microglia. Neuro Oncol 12: 1113-1125, 2010.

26. Straussman R, Morikawa T, Shee K, et al: Tumour microenvironment elicits innate resistance to RAF inhibitors through HGF secretion. Nature 487: 500-504, 2012.

27. Borovski T, Verhoeff JJC, ten Cate R, et al: Tumor microvascular supports proliferation and expansion of glioma-propagating cells. In J Cancer 125: 1222-1230, 2009.

28. Gazdzinski LM and Nieman BJ: Cellular imaging and texture analysis distinguish differences in cellular dynamics in mouse brain tumors. Magn Reson Med: May 9, 2013. doi: 10.1002/ mrm.24790. 